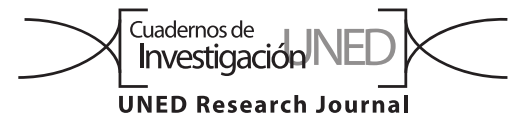

\title{
Tiempo y dosis óptimas de fluoximesterona, para masculinización de crías del pez maya Cichlasoma urophthalmus (Percidae: Cichlidae)
}

\author{
Irma Gutiérrez-Sigueros', Carlos A. Cuenca-Soria', Leonardo I. Navarro-Angulo', Emilio J. Maldonado-Enríquez', \\ Carolina E. Melgar-Valdes ${ }^{1}$ \& Alfonso Castillo-Domínguez ${ }^{1}$
}

1. Universidad Juárez Autónoma de Tabasco. División Académica Multidisciplinaria de los Ríos. Carretera Tenosique-Estapilla km 1, Colonia

Solidaridad. Tenosique, Tabasco, México.86901; sigeros01@gmail.com, cccp0900@hotmail.com, leonardo.navarro@ujat.mx, emi_maen78@hotmail.com, cemv81@gmail.com, alfonsin75@gmail.com

Recibido 09-IV-2018 • Corregido 09-VI-2018 • Aceptado 14-VI-2018

\begin{abstract}
Optimal time and dose of fluoxymesterone, for masculinization of Mayan fish Cichlasoma urophthalmus (Percidae: Cichlidae). The production of all-male populations in cichlid fish, is crucial to obtain high yielding in fish farming. The aim of this paper was to determinate the optimal dose and time of exposure to fluoxymesterone (FM), during the masculinization of Mayan cichlid Cichlasoma urophthalmus larvae. Therefore, two periods of exposure to FM (45 and 60 days) and four androgen doses $(0,15,30$ and $60 \mathrm{mg} / \mathrm{kg}$ of food) were tested, having a total of five treatments with FM $(15 \mathrm{mg} 60 \mathrm{~d}, 30 \mathrm{mg} 45 \mathrm{~d}$, $30 \mathrm{mg} 60 \mathrm{~d}$, $60 \mathrm{mg} 45 \mathrm{~d}$ and $60 \mathrm{mg} 60 \mathrm{~d}$ ) and one control group (0mg 60d, CTL), in triplicate. One kilogram of Purina tilapia starter feed (45\% protein) was sprayed with a hormone solution previously dissolved in ethyl alcohol, according to each separate testing dose mentioned above. $C$. urophthalmus larvae were fed hormonal foods ad libitum (seven days after hatching) based on each treatment. Results revealed that male average rate $(69,1 \pm 2,7 \%)$ obtained from the $60 \mathrm{mg} / \mathrm{kg}$ of FM dose, administered with food for 45 days (60mg $45 \mathrm{~d}$ ) was significantly greater in comparison to the other tested treatments (Tukey, $\mathrm{p}<0,05, \mathrm{k}=6, \mathrm{~N}-\mathrm{k}=$ 12). Thus, it is necessary to review new techniques to administer steroid hormones (bioencapsulation) that, on one hand, reduce precocious reproduction problems in the cultivation of this species and, on the other hand, establish more environmentally friendly techniques, which could minimize the presence of steroid hormone waste on aquatic ecosystems.
\end{abstract}

Key words: Time, dose, fluoxymesterone, masculinization, Cichlasoma, urophthalmus.
RESUMEN: La masculinización en peces cíclidos, es de importancia capital para fines de mejora en la producción piscícola. El objeto de la presente investigación fue obtener la dosis y tiempo óptimos de exposición a la hormona fluoximesterona (FM), durante la masculinización del cíclido Maya Cichlasoma urophthalmus. Así, se probaron, dos tiempos de exposición (45 y 60 días) y cuatro dosis de andrógeno (0, 15,30 y $60 \mathrm{mg} / \mathrm{kg}$ de alimento), de manera que se tuvieron cinco tratamientos (15mg 60d, 30mg 45d, 30mg 60d, 60mg $45 \mathrm{~d}$ y $60 \mathrm{mg} 60 \mathrm{~d}$ ) y un grupo testigo sin hormona ( $0 \mathrm{mg} 60 \mathrm{~d}, \mathrm{CTL}$ ), por triplicado. Un kilogramo de alimento iniciador para tilapia Purina (45\% de proteína), fue rociado con solución hormonada previamente disuelta en alcohol etílico, a razón de las dosis de prueba antes mencionadas por separado. Se administró alimento hormonado a saciedad, a crías de C. urophthalmus (siete días después de la eclosión) según el tratamiento. Los resultados revelaron que la proporción promedio de machos $(69,1 \pm 2,7 \%)$, obtenida con la dosis $60 \mathrm{mg} / \mathrm{kg}$ de $\mathrm{FM}$, suministrada vía alimento por 45 días $(60 \mathrm{mg} 45 \mathrm{~d}$ ), fue significativamente mayor, al resto de los tratamientos probados (Tukey, $\mathrm{p}<0,05, \mathrm{k}=6, \mathrm{~N}-\mathrm{k}=12$ ). Tales resultados obligan a revisar nuevas técnicas para la administración de hormonas esteroides (bioencapsulación), que por un lado, disminuyan los problemas de reproducción precoz en el cultivo de esta especie nativa, y por otro, constituyan técnicas más amigables con el entorno ambiental, lo cual podría minimizar la carga residual de hormonas esteroides en los cuerpos acuáticos.

Palabras clave: Tiempo, dosis, fluoximesterona, masculinización, Cichlasoma, urophthalmus.
En el Sureste de México, existen especies nativas diversas, con amplio potencial de cultivo. El grupo de los cíclidos, es uno de los grupos promisorios al respecto. Sin embargo, una de las características reproductivas de los peces cíclidos, es su reproducción precoz, ya que aproximadamente a los tres meses de edad pueden alcanzar la madurez sexual. Esto es característico de tilapia
Oreochromis niloticus y otros cíclidos (Hurtado, 2005), lo cual genera problemas para obtener en un tiempo adecuado tallas homogéneas aceptables en el mercado (Martínez-Palacios \& Chávez, 1994). Para solventar estos inconvenientes se emplean diversas técnicas para la producción de poblaciones monosexo. Tales poblaciones, ofrecen diferentes beneficios, incluyendo un crecimiento 
rápido y el control de la reproducción no deseada, evitando de este modo el desvío de energía a la producción de gametos, y promoviendo el incremento de la masa muscular y talla. Con ello se evita a su vez, que los tiempos de cultivo puedan prolongarse forma considerable (Green et al., 1997)

Por otro lado, la mojarra latinoamericana o cíclido Maya C. urophthalmus, es una de las mojarras nativas del sureste mexicano más ampliamente estudiada, con fines de cultivo (Martínez-Palacios \& Ross, 1994; Pérez-Sánchez \& Páramo-Delgadillo, 2008). Esta especie tiene una tasa de fecundidad de 2000 a 4000 huevos por desove, permitiendo una buena producción de cría en comparación al de las tilapias (Morales, 1991). Así mismo, tiene una reproducción frecuente todo el año, llegándose a reproducir a una talla mínima de $12 \mathrm{~cm}$ y $50 \mathrm{~g}$ (Martínez-Palacios \& Ross, 1988). La elevada tasa reproductiva de C. urophthalmus, genera un gran número de crías, dando como resultado, sobrepoblación en los estanques. Esto disminuye los rendimientos y encarece los costos de producción. Para mejorar la rentabilidad económica y financiera en los cultivos de cíclidos, es indispensable manejar poblaciones monosexo debido a que los machos alcanzan mayores tallas que las hembras (Hurtado, 2005)

Existe un amplio número de trabajos publicados, en lo que concierne a especies, técnicas de administración hormonal androgénicas/estrogénicas, dosis, condiciones de cultivo, entre otros Jiménez-Badillo y ArredondoFigueroa (2000a, 2000b); Contreras-Sánchez (2003); Hurtado (2005); López, Carvajal y Botero (2007); VidalLópez et al., (2009); Liñan-Cabello, Robles-Basto y MenaHerrera (2012). Dentro de las técnicas de masculinización destaca, la técnica de administración de hormonas androgénicas en el alimento, durante las etapas tempranas de su desarrollo, cuando aún no se diferencian los tejidos gonádicos, de acuerdo a Jiménez-Badillo y ArredondoFigueroa (2000a). Para que el proceso de masculinización se lleve a cabo de manera exitosa, la hormona debe administrarse en el período lábil, es decir, justo antes del inicio de la diferenciación sexual (Hunter \& Donaldson, 1983). En los peces cíclidos como la tilapia, el período lábil se ha detectado entre los 11 y 21 días después de la fertilización (Popma \& Green, 1990)

En algunos peces el desarrollo de las gónadas es afectado por la exposición a esteroides antes o después del período de diferenciación sexual; siendo la hormona 17-a-metiltestosterona el esteroide sintético más ampliamente utilizado para estos fines, cuya potencialidad, ha sido ensayada en distintas especies (Jiménez-Badillo y Arredondo-Figueroa (2000a); Alfonso y Wassermann (2002); Delvin y Nagahama, 2002). No obstante, la poca disponibilidad de la 17-a-metiltestosterona en el sureste de México, encarece los costos de su uso. La masculinización de peces cíclidos como $C$. urophthalmus, haciendo uso de la hormona androgénica FM, puede presentar ventajas diversas, por su bajo costo y fácil acceso en el sureste de México. Así mismo, la mayor cantidad de dosis de hormona es metabolizada y eliminada antes de que el pez alcance su talla comercial, donde el hígado convierte el compuesto androgénico, en sustancias más solubles que al final, son eliminadas vía orina y bilis (Marcillo \& Landivar, 2000)

Debido a los beneficios antes expuestos, con relación a la utilización de hormonas androgénicas (como la FM, con sus ventajas inherentes), así como las implicaciones negativas que conllevan los problemas de reproducción precoz en peces cíclidos como C. urophthalmus y por ende, las afectaciones en la producción; es que el objeto del presente trabajo fue evaluar la dosis y tiempos óptimos de aplicación de la hormona fluoximesterona, para masculinizar crías de mojarra castarrica C. urophthalmus, en la región sureste de México

\section{MATERIALES Y MÉTODOS}

El presente estudio se llevó a cabo en las instalaciones experimentales del área de acuacultura, Universidad Juárez Autónoma de Tabasco, División Académica Multidisciplinaria de los Ríos (DAMRÍOS), ubicada en el kilómetro 1, carretera Tenosique-Estapilla, Tabasco, México. Las larvas de $C$. urophthalmus fueron obtenidas a partir del lote de reproductores (3:1, hembras-macho), confinados en tinas de plástico circulares $\left(1,7 \mathrm{~m}^{3}\right)$, en un sistema de recirculación abierto. A los 7 días posteriores a la eclosión las crías fueron trasladadas del sistema de reproducción al sistema experimental, equipado con una bomba de 1,5hp (A.O. Smith ${ }^{\circ}$ y un filtro de arena (Series Competition ${ }^{\circ}$ ), donde fueron contadas y confinadas a razón de 30 organismos por cada tina de $200 \mathrm{~L}$.

El diseño experimental consistió en probar, por un lado, dos tiempos de exposición (45 y 60 días) y por otro, cuatro dosis de andrógeno $(0,15,30$ y $60 \mathrm{mg} / \mathrm{kg}$ de alimento obteniendo así cinco tratamientos $(15 \mathrm{mg} 60 \mathrm{~d}$, $30 \mathrm{mg} 45 \mathrm{~d}$, $30 \mathrm{mg} 60 \mathrm{~d}, 60 \mathrm{mg} 45 \mathrm{~d}$ y $60 \mathrm{mg} 60 \mathrm{~d}$ ), así como un grupo testigo sin FM (0mg 60d, CTL). Todos los tratamientos fueron evaluados por triplicado, donde fueron confinados 60 peces por tratamiento. Los parámetros físicos y químicos fueron monitoreados diariamente con un termómetro (Brannan USA), un oxímetro (YSI 55, California USA) y un potenciómetro (Denver Instrument UB-10, Denver, Colorado, USA) 
El alimento hormonado se elaboró según JiménezBadillo y Arredondo-Figueroa (2000b), utilizando 4kg de alimento comercial Nutripec, Purina (45\% proteína), después fueron molidos finamente, en un molino para café, (Nixtamatic, NTC-01) de 1/3hp. El alimento en polvo fue cribado, en un tamiz del número 50 . Por otro lado, tabletas de FM, con ingrediente activo 2,5mg, fueron trituradas en un mortero de porcelana, hasta obtener un polvo fino, y se disolvió en $500 \mathrm{ml}$ de alcohol etílico absoluto (99,98\% de pureza, J. T. Baker), para obtener las dosis de hormona, por kilogramo de alimento deseadas.

A las larvas de $C$. urophthalmus, se les suministró el alimento hormonado de acuerdo al diseño experimental planteado; a la par de un tratamiento de larvas, con alimento sin hormona. La alimentación de organismos fue ad libitum diariamente aplicando cuatro raciones al día a intervalos de $2 \mathrm{~h}$. Una vez concluidos los tratamientos hormonados, las crías fueron transferidas a tinas del mismo volumen de agua que las del experimento, las cuales a su vez, forman parte de un sistema de recirculación continuo. En esta fase de crecimiento, a los peces se les siguió administrando el mismo alimento comercial libre de hormona, hasta llevarlos a una talla promedio de $7 \mathrm{~cm}$ de longitud total.

Previo a la disección de gónadas, los organismos fueron sacrificados mediante una sobredosis del anestésico MS222 (tricaine methanesulphonate, Argent Laboratorios, Redmont, USA). Posteriormente, se procedió a la identificación del sexo, mediante la técnica del Squash propuesta por Jiménez-Badillo y Arredondo-Figueroa (2000b), la cual consiste en extraer y colocar las gónadas sobre un portaobjeto, para luego adicionarle colorante de Wright ${ }^{\circ}$ para ser teñidas, seguidamente se ejerce una leve presión con el cubreobjetos para aplastar el tejido gonádico. Terminado el proceso de las preparaciones se observaron en el microscopio óptico (Labomed) a 10x, 40x y $100 x$ de aumento, para determinar el sexo de los peces de acuerdo a las características histológicas.

Con lo que respecta a las pruebas estadísticas empleadas para el análisis de los datos, los porcentajes de masculinización por tratamiento, fueron sujetos a una transformación como sugiere Zar (1974), de modo que los datos expresados en valores porcentuales, tiendan a la normalidad y homocedasticidad. Para tal fin, se empleó la transformación propuesta por Cuenca et al., (2013). Se empleó el programa Statistica ${ }^{\mathrm{TM}}$ versión 8.0, para las pruebas de normalidad, homocedasticidad, ANOVA y post hoc (Tukey).

\section{RESULTADOS}

Los resultados de la masculinización de crías de $C$. urophthalmus, por efecto de la FM, se pueden apreciar en el cuadro 1. Los porcentajes de masculinización obtenido por los distintos tratamientos, fueron significativos (Tukey, $p<0,05, k=6, N-k=12$ ); siendo el tratamiento $60 \mathrm{mg} / \mathrm{kg}$ de FM por 45 días ( $60 \mathrm{mg} 45 \mathrm{~d}$ ), con el mayor porcentaje de masculinización observado $(69,1 \pm 2,7 \%)$; en tanto que el tratamiento $60 \mathrm{mg} 60 \mathrm{~d}$, tuvo el menor rendimiento de machos $(22 \pm 3,1 \%)$. Así mismo, el tratamiento control (0mg 60d), obtuvo el 31,3 $36,3 \%$ de peces macho.

Por otro lado, los parámetros de crecimiento y de supervivencia de crías de $C$. urophthalmus, por efecto de los distintos tratamientos, se pueden visualizar también en el cuadro 1. Los parámetros finales de crecimiento,

CUADRO 1

Parámetros de crecimiento, supervivencia y porcentaje de masculinización, por efecto de los distintos tratamientos"

$\begin{array}{lccccrr} & \text { CTL } & 15 \mathrm{mg} \mathrm{60d} & 30 \mathrm{mg} 45 \mathrm{~d} & 30 \mathrm{mg} \mathrm{60d} & 60 \mathrm{mg} \mathrm{45d} & 60 \mathrm{mg} \mathrm{60d} \\ \text { W }_{\mathrm{o}}(\mathrm{g}) & 0,01 \pm 0,0^{\mathrm{a}} & 0,01 \pm 0,0^{\mathrm{a}} & 0,01 \pm 0,0^{\mathrm{a}} & 0,01 \pm 0,0^{\mathrm{a}} & 0,01 \pm 0,0^{\mathrm{a}} & 0,01 \pm 0,0^{\mathrm{a}} \\ \text { W (g) } & 0,21 \pm 0,06^{\mathrm{bc}} & 0,44 \pm 0,11^{\mathrm{ab}} & 0,35 \pm 0,07^{\mathrm{abc}} & 0,55 \pm 0,14^{\mathrm{a}} & 0,15 \pm 0,05^{\mathrm{c}} & 0,56 \pm 0,09^{\mathrm{a}} \\ \text { L (mm) } & 21,4 \pm 2,8^{\mathrm{a}} & 26,9 \pm 3,8^{\mathrm{a}} & 25,6 \pm 2,9^{\mathrm{a}} & 28,2 \pm 1,2^{\mathrm{a}} & 19,0 \pm .1,1^{\mathrm{a}} & 28,5 \pm 2,0^{\mathrm{a}} \\ \text { TCE (\%) } & 4,65 \pm 0,15^{\mathrm{b}} & 5,71 \pm 0,47^{\mathrm{a}} & 5,25 \pm 0,3^{\mathrm{a}} & 5,86 \pm 0,46^{\mathrm{a}} & 3,92 \pm 0,6^{\mathrm{b}} & 6,01 \pm 0,26^{\mathrm{a}} \\ \text { WG/d (g) } & 0,003 \pm 0,0^{\mathrm{bc}} & 0,007 \pm 0,0^{\mathrm{ab}} & 0,006 \pm 0,0^{\mathrm{bc}} & 0,009 \pm 0,0^{\mathrm{a}} & 0,002 \pm 0,0^{\mathrm{c}} & 0,009 \pm 0,0^{\mathrm{a}} \\ \text { FC } & 2,33 \pm 0,79^{\mathrm{a}} & 2,34 \pm 0,62^{\mathrm{a}} & 2,16 \pm 0,47^{\mathrm{a}} & 2,52 \pm 0,69^{\mathrm{a}} & 2,25 \pm 0,66^{\mathrm{a}} & 91,6 \pm 2,4^{\mathrm{a}} \\ \text { S (\%) } & 91,3 \pm 2,2^{\mathrm{a}} & 94,1 \pm 1,7^{\mathrm{a}} & 90,5 \pm 3,4^{\mathrm{a}} & 93,4 \pm 1.9^{\mathrm{a}} & 92,7 \pm 3,2^{\mathrm{a}} & 91,6 \pm 2,4^{\mathrm{a}} \\ \text { M (\%) } & 31,3 \pm 6,3^{\mathrm{c}} & 25,9 \pm 1,6^{\mathrm{c}} & 41,9 \pm 3,8^{\mathrm{b}} & 26,2 \pm 4,6^{\mathrm{c}} & 69,1 \pm 2,7^{\mathrm{a}} & 22,0 \pm 3,1^{\mathrm{c}}\end{array}$

1/ (CTL: Alimento comercial sin fluoximesterona; 15mg 60d: tratamiento con 15mg/kg (fluoximesterona/alimento), durante 60 días (y sucesivamente para el resto de los tratamientos)

2/ Índice de siglas: $\mathrm{W}_{\mathrm{o}}=$ Peso inicial; $\mathrm{W}=$ Peso final $(60 \text { días })^{*} ; \mathrm{L}=$ Longitud total final; TCE = Tasa de crecimiento específica; $W G / d=$ Peso ganado al día; FC = Factor de conversión; $\mathrm{S}=$ Porcentaje de supervivencia; $\mathrm{M}=$ Porcentaje de masculinización. Letras distintas en superíndices indican diferencias significativas entre tratamientos. 
CUADRO 2

Parámetros físicos y químicos, durante la masculinización de $C$. urophthalmus.

\begin{tabular}{lccccc}
\multicolumn{1}{c}{ Parámetro } & $\mathrm{t}_{0}$ & $\mathrm{t}_{15}$ & $\mathrm{t}_{30}$ & $\mathrm{t}_{45}$ & $\mathrm{t}_{60}$ \\
Temperatura $\left({ }^{\circ} \mathrm{C}\right)$ & $29 \pm 0,55$ & $27 \pm 0,45$ & $28 \pm 0,55$ & $29 \pm 2,07$ & $28 \pm 1,86$ \\
Oxígeno disuelto $(\mathrm{mg} / \mathrm{L})$ & $5,1 \pm 0,19$ & $5,3 \pm 0,28$ & $6,1 \pm 0,14$ & $5,9 \pm 0,44$ & $5,76 \pm 0,76$ \\
$\mathrm{pH}$ & $7,52 \pm 0.36$ & $7,43 \pm 0.22$ & $7,33 \pm 0.08$ & $7,27 \pm 0.28$ & $7,21 \pm 0,18$ \\
\hline
\end{tabular}

el peso final (W), el peso ganado-día final (WG/d), y la tasa de crecimiento específica (TCE), fueron significativos (Tukey, $\mathrm{p}<0,05, \mathrm{k}=6, \mathrm{~N}-\mathrm{k}=12$ ).

Los valores máximos finales de cada parámetro (W, WG/d, TCE), fueron 0,56 $\pm 0,09 \mathrm{~g}, 0,009 \pm 0,0 \mathrm{~g} /$ día y $6,01 \%$, respectivamente; mientras que los valores menores de estos mismos parámetros, correspondieron al tratamiento $60 \mathrm{mg} 45 \mathrm{~d}$, con $0,15 \pm 0,05 \mathrm{~g}, 0,002 \pm 0,0$ y $3,92 \pm 0,6 \%$. Los factores de condición ( $F C$ ) finales, no fueron estadísticamente significativos $(p>0,05)$; mostrando fluctuaciones alrededor de 2,5 , entre tratamientos. Sin embargo, el tratamiento que presentó el mayor factor de condición fue el $30 \mathrm{mg} 60 \mathrm{~d}(2,52 \pm 0,69)$, mientras que el menor, se obtuvo por efecto del tratamiento $30 \mathrm{mg} 45 \mathrm{~d}(2,16 \pm 0,47)$. Paralelamente, la supervivencia no observó diferencias significativas entre los distintos tratamientos ( $p>0,05)$, situándose por arriba del $90 \%$ en todos los casos. Así mismo en el cuadro 2, se muestran los valores medios de parámetros de la calidad del agua (temperatura, oxígeno disuelto y $\mathrm{pH}$ ) monitoreados, a lo largo del experimento.

\section{DISCUSIÓN}

Los porcentajes obtenidos en la presente investigación, muestran que el tratamiento con 60mg de FM, aplicado por 45 días, arrojó el mayor rendimiento, en cuanto al número de machos producidos $(69,1 \pm 2,7 \%)$. Este resultado, sin embargo, es menor al número de machos $(80,6 \%)$, obtenidos por Jiménez-Badillo y ArredondoFigueroa, (2000a), cuando aplicaron una dosis de 1,0mg de FM a crías de tilapia O. niloticus, por 30 días. Los mismos autores reportaron un $92 \%$ de machos, aplicando una dosis de $40 \mathrm{mg}$ de 17 -a-metiltestosterona, siendo un valor porcentual mucho mayor al de la presente investigación. Moreno, Rodríguez, Barriga y Arredondo (2003), hallaron resultados más promisorios, alcanzando un $95 \%$ de masculinización de $O$. niloticus con $\mathrm{FM}$, a razón de $5 \mathrm{mg} / \mathrm{kg}$ (hormona/alimento), durante 35 días. Sin embargo, López et al., (2007) reportaron porcentajes de masculinización del 100 y 92,6\%, en crías de tilapia roja tratada vía oral y por inmersión con 17-a-metiltestosterona. Las dosis empleadas fueron $60 \mathrm{mg} / \mathrm{kg}$, (hormona/alimento), y $1,8 \mathrm{mg} / \mathrm{L}$ (hormona/agua), respectivamente.

La utilización de la FM como hormona masculinizante, dispone de ciertas ventajas en el Sureste de México, debido a su bajo costo y amplia disponibilidad en el mercado. Por otro lado, aunque se han comparado distintas especies, condiciones de cultivo, tipo de hormona, tiempos de exposición androgénica y técnicas de administración; la hormona esteroide FM, no tuvo los resultados que se pudieran traducir en la obtención de poblaciones monosexo masculinas, por arriba del $90 \%$ de efectividad. No al menos con crías de $C$. urophthalmus, y técnica de administración empleada en la presente investigación. A este respecto, Contreras-Sánchez et al. (2003), reportaron valores porcentuales cercanos al 95\% de masculinización, en crías de la misma especie, tratada con 17-a-metiltestosterona $(15,30,45$ y $60 \mathrm{mg} / \mathrm{kg}$, hormona/alimento). Los mismos autores encontraron un porcentaje de machos por arriba del 95\%, al alimentar crías de C. urophthalmus con 17-a-metiltestosterona, bioencapsulada en nauplios de Artemia, durante 60 días de tratamiento.

Por lo que es posible enfatizar, la importancia de seguir llevando a cabo mayores estudios en crías de C. urophthalmus, sobre todo si se proponen nuevas técnicas de administración de FM, como sugieren Contreras-Sánchez et al. (2003), López et al. (2007) y Vidal-López et al. (2009), probando con la inmersión y bioencapsulación, respectivamente. Adicionalmente, estas técnicas pudieran minimizar los efectos negativos, que la presencia de este tipo de sustancias implica, en el medio ambiente acuático. Más aún, cabe hacer notar la dificultad de dilución de las tabletas de Stenox ${ }^{\circledR}$ (nombre comercial de la FM) en etanol absoluto, lo que posiblemente puede mermar la cantidad de FM presente en la solución hormonada a incorporar en el alimento. Lo anterior puede significar otra desventaja adicional, de la técnica de administración oral de hormonas esteroides, como subrayan López et al. (2007)

En lo que respecta a la supervivencia en la presente investigación, ésta mostró valores por arriba del 90\% en todos los tratamientos, estando dentro de los intervalos de supervivencia reportados por Liñán Cabello et al. (2012) 
e incluso mayores a los obtenidos por Jiménez-Badillo y Arredondo-Figueroa (2000a); López et al. (2007) y VidalLópez et al. (2009), quienes reportaron porcentajes de supervivencia por debajo del $90 \%$. En lo que concierne a los parámetros de crecimiento, y siguiendo con JiménezBadillo y Arredondo-Figueroa (2000a), los pesos finales que obtuvieron en crías de tilapia, resultaron superiores a los encontrados en el presente trabajo, para crías de C. urophthalmus. En tanto que los valores finales del factor de condición, resultado de las investigaciones de Liñán-Cabello et al. (2012), para crías de tilapia del Nilo O. niloticus, tratadas con 17-a-metiltestosterona e inyectadas con hormona de crecimiento, resultaron cercanos a los hallados en C. urophthalmus. Nehemia, Maganira y Rumisha (2012) reportaron factores de condición menores que la unidad, para Tilapia zilli y Oreochromis urolepis urolepis, en condiciones marinas (35\%o); mientras que para las mismas especies, encontraron factores de condición entre 0,86 y 2,07 en condiciones de agua dulce. A reserva de que es necesario realizar más investigaciones, los factores de condición relativamente altos para C. urophthalmus (entre 2 y 2,5 ), pueden ser atribuibles a las condiciones de agua dulce del cultivo experimental, en el presente estudio. Factores de condición entre 2,63 y 3,4 fueron reportados por Mossad (1990), para T. zilli, cultivada en agua dulce. Sin embargo, estas diferencias pueden ser también atribuibles a factores relacionados con la biología de la especie como el desarrollo gonádico. El factor de condición de los peces puede verse afectado por diversos factores, tales como el estrés, desarrollo gonádico, calidad del agua, estacionalidad y disponibilidad de alimento (Khallaf, Galal \& Athuman, 2003). Aunque con relación a estos dos últimos factores, cabe mencionar que los peces bajo los distintos tratamientos fueron alimentados a saciedad y a su vez, afectados por los mismos cambios de estación. Por consiguiente, las diferencias del factor de condición entre grupos, puede considerarse despreciable.

Con relación a posibles efectos anabólicos, por el uso de hormonas esteroides en piscicultura, Jiménez-Badillo y Arredondo-Figueroa (2000a) reportaron que la tasa de crecimiento específica en crías de tilapia, bajo efecto de un tratamiento con 17-a-metiltestosterona, resultó mayormente significativo (con relación a un tratamiento sin hormona). De igual manera, Liñán-Cabello et al. (2012), obtuvieron un mejor desenvolvimiento de los parámetros de crecimiento, en tilapias tratadas con 17-a-metiltestosterona y hormona del crecimiento. Así mismo, Salgado et al. (2008) encontraron que la hormona androgénica acetato de trembolona, tuvo un efecto anabólico en juveniles del pez de ornato Poecilia reticulata, al ser tratados con alimento hormonado, a razón de $300 \mathrm{mg} /$ kg de alimento durante 60 días. Sin embargo, los parámetros de crecimiento evaluados en la presente investigación, muestran resultados contradictorios, dado que, aunque el tratamiento $60 \mathrm{mg}$ de FM por 45 días, mostró mayor número de machos revertidos, también fue el que observó el menor peso y tasa de crecimiento específica al final, respecto de los demás tratamientos, incluido el tratamiento testigo sin hormona.

No obstante, C. urophthalmus es una especie nativa que ha sido ampliamente estudiada. Es una especie altamente demandada en el mercado local, con una alta adaptabilidad en cautiverio, fertilidad y supervivencia, lo que representa amplias ventajas para su cultivo intensivo (Pérez-Sánchez y Páramo-Delgadillo, 2008). Por consiguiente, es menester continuar los estudios acerca del empleo de hormonas androgénicas, con el fin de realizar mejoras a la masculinización de $C$. urophthalmus, y de este modo, se pueda contribuir a sentar las bases técnicas de su cultivo.

\section{AGRADECIMIENTOS}

El presente trabajo de investigación, fue posible gracias al financiamiento del Consejo Nacional de Ciencia y Tecnología de México, número de propuesta 16472; así como a colaboradores para la realización del proyecto.

\section{REFERENCIAS}

Alfonso, L. O. B., \& Wassermann, G. J. (2002). Immersion in an aromatizable and non- aromatizable androgens induces high rates of masculinization in Nile Tilapia Oreochromis niloticus. In Book of abstracts: Aquaculture América (pp. 2). California USA.

Contreras-Sánchez, W. M., Márquez-Couturier, G., Feist, G. W., Hernández-Franyutti, A., Schreck, C. B., \& Giannico, G. (2003). Diversification of Aquacultural Practices by Incorporation of Native species and Implementation of Sex Invertion Techniques. (Technical Report). Aquaculture CRSP, 31, 189-195.

Cuenca, C. A., Álvarez-González, C. A., Ortiz-Galindo, J. L., Guerrero-Zárate, R., Perera-García, M. A., HernándezGómez, R. E., \& Nolasco-Soria, H. (2013). Digestibilidad in vitro de ingredientes proteínicos en la mojarra castarrica Cichlasoma urophthalmus. Ecosistemas y Recursos Agropecuarios, 29(3), 263-275.

Devlin, R. H., \& Nagahama, Y. (2002). Sex determination and sex differentiation on fish: an overview genetic, physiological and environmental influences. Aquaculture, 208, 191364. doi: 10.1016/S0044-8486(02)00057-1

Green, B. W., Veverica, K. L., \& Fitzpatrick, M. S. (1997). Fry and fingerling production. In Hillary, S., Egna \& Claude, E., 
Boyd (Eds.), Dynamics of Pond Aquacuture (pp. 437). New York, USA: CRC Press.

Hunter, G. A., \& Donaldson, E. M. (1983). Hormonal sex control and its application to fish aquaculture. In W. S. Hoar., D. J. Randall \& E.M. Donaldson (Eds.), Fish physiology (pp. 223-303). Vol. IXB. New York/London: Academic press.

Hurtado, T. N. (2005). Inversión sexual en tilapias: revisión bibliográfica. Recuperado de http://www.revistaaquatic. com/documentos/docs/nh_invsextilapia.pdf

Jiménez-Badillo, M. L., \& Arredondo-Figueroa, J. L. (2000a). Effect of oral treatment of synthetic androgens on sex ratio, survival and growth rates, in three strains of tilapia. Hidrobiológica, 10(2), 115-120.

Jiménez, B. M. L., \& Arredondo, J. L. (2000b). Manual técnico para la reversión sexual de tilapia. Serie Desarrollo Tecnológicos en Acuacultura. México: Editorial de la Universidad Autónoma Metropolitana Unidad Iztapalapa.

Khallaf, E., Galal, M., \& Athuman, M. (2003). The biology of Oreochromis niloticus in a polluted canal. Ecotoxicology, 12, 405-416. doi: 10.1023/A:1026156222685

Liñan-Cabello, M. A., Robles-Basto, C. M., \& Mena-Herrera A. (2012). Somatic growth effects of intramuscular injection of growth hormone in androgen-treated juvenile Nile tilapia, Oreochromis niloticus (Perciformes: Cichlidae). Revista de Biología Tropical, 61(1), 203-212.

López, C. A., Carvajal D. L., \& Botero, M. C. (2007). Masculinización de tilapia roja Oreochromis niloticus por inmersión, utilizando 17 a-metiltestosterona. Revista Colombiana de Ciencias Pecuarias, 20, 318-326.

Marcillo, G. E., \& Landivar, Z. J. (2000). Tecnología de producción de alevinos monosexo de tilapia. Ecuador: Editorial de la Escuela Superior Politécnica del Litoral. Recuperado de http://www.dspace.espol.edu.ec/xmlui/ handle/123456789/6342

Martínez-Palacios, C. A., \& Ross L. G. (1988). The feeding ecology of the Central American cichlid Cichlasoma urophthalmus (Günther 1862). Journal of Fish Biology, 33, 665-670. doi: 10.1111/j.1095-8649.1988.tb05512.x
Martínez-Palacios, C. A., \& Ross, L. G. (1994). Biología y cultivo de la mojarra latinoamericana Cichlasoma urophthalmus (1 ${ }^{\text {a }}$ ed.). CONACyT México: Grafos Editores S. A de C. V.

Morales-Díaz, A. (1991). La tilapia en México. Biología, cultivo y pesquerías. México: AGT Editor.

Moreno, E. A., Rodríguez, C. A., Barriga, S. I., \& Arredondo, J. L. (2003). Producción de Tilapia del Nilo (Oreochromis niloticus) masculinizada con la hormona fluoximesterona en sistemas de recirculación. En Anónimo, (Ed.), Memorias del Congreso Iberoamericano Virtual de Acuacultura (pp. 77-88). Zaragoza, España.

Mossad, M. N. M. (1990). Biological studies on five fish species from Lake Qarun, Egypt, Length-weight relationship and condition factor. Journal of Zoological Society, 21, 331-344.

Nehemia, A., Maganira, J. D., \& Rumisha, C. (2012). LengthWeight relationship and condition factor of tilapia species grown in marine and fresh water ponds. Agriculture and Biology Journal of North America, 3(3), 117-124. doi: 10.5251/abjna.2012.3.3.117.124

Pérez-Sánchez, E., \& Páramo-Delgadillo, S. (2008). The culture of cichlids ofthesoutheasternMexico.AquacultureResearch, 39: 777-783. doi: 10.1111/j.1365-2109.2008.01929.x

Popma, T. J., \& Green, W. B. (1990). Aquaculture production manual: Sex reversal of tilapia in earthen ponds. Research and development. AL, USA: International Center for aquaculture. Alabama Agricultural Experiment Station, Serie 35. Aurburn University.

Salgado, Z. H., Azpeitia, H. A., Marañón, H. S., \& Maya, P. E. (2008). Efecto anabólico y androgénico del esteroide acetato de trembolona en el guppy Poecilia reticulata. Veterinaria México, 39(3), 269-279.

Vidal-López J. M., Álvarez-González, C. A., Contreras-Sánchez, W. M., \& Hernández-Vidal, U. (2009). Masculinización del cíclido nativo Tenhuayaca, Petenia splendida. (Günther, 1862), usando nauplios de Artemia como vehículo del esteroide 17-a metiltestosterona. Hidrobiológica, 19(3), 211-216.

Zar, J. H. (1974). Biostatistical Analysis. New Jersey, USA: PrenticeHall, Englewood Clifs. 